Review

\title{
Carotenoid $\beta$-Ring Hydroxylase and Ketolase from Marine Bacteria-Promiscuous Enzymes for Synthesizing Functional Xanthophylls
}

\section{Norihiko Misawa}

Research Institute for Bioresources and Biotechnology, Ishikawa Prefectural University, Suematsu, Nonoichi-machi, Ishikawa 921-8836, Japan; E-Mail: n-misawa@ishikawa-pu.ac.jp;

Tel.: +81-76-227-7525; Fax: +81-76-227-7557

Received: 21 March 2011; in revised form: 19 April 2011 / Accepted: 26 April 2011 /

Published: 6 May 2011

\begin{abstract}
Marine bacteria belonging to genera Paracoccus and Brevundimonas of the $\alpha$-Proteobacteria class can produce $\mathrm{C}_{40}$-type dicyclic carotenoids containing two $\beta$-end groups ( $\beta$ rings) that are modified with keto and hydroxyl groups. These bacteria produce astaxanthin, adonixanthin, and their derivatives, which are ketolated by carotenoid $\beta$-ring 4(4')-ketolase (4(4')-oxygenase; $\mathrm{CrtW}$ ) and hydroxylated by carotenoid $\beta$-ring 3(3')-hydroxylase (CrtZ). In addition, the genus Brevundimonas possesses a gene for carotenoid $\beta$-ring $2\left(2^{\prime}\right)$-hydroxylase $(\mathrm{CrtG})$. This review focuses on these carotenoid $\beta$-ring-modifying enzymes that are promiscuous for carotenoid substrates, and pathway engineering for the production of xanthophylls (oxygen-containing carotenoids) in Escherichia coli, using these enzyme genes. Such pathway engineering researches are performed towards efficient production not only of commercially important xanthophylls such as astaxanthin, but also of xanthophylls minor in nature (e.g., $\beta$-ring(s)-2(2')-hydroxylated carotenoids).
\end{abstract}

Keywords: Paracoccus; Brevundimonas; marine bacteria; ketocarotenoid; functional xanthophyll

\section{Introduction}

Many bacteria that have been isolated from marine environments can synthesize a variety of carotenoid pigments [1]. For example, acyclic $\mathrm{C}_{30}$-type carotenoic acids were identified in some marine bacteria such as Planococcus maritimus [2] and Rubritalea squalenifaciens [3]. Algoriphagus sp. 
KK10202C of the Flexibacteraceae family, which was isolated from a marine sponge, was found to produce flexixanthin (( $3 S)$-3, $1^{\prime}$-dihydroxy-3',4'-didehydro- $1^{\prime} 2^{\prime}$-dihydro- $\beta, \psi$-caroten-4-one) and deoxyflexixanthin (1'-hydroxy-3',4'-didehydro- $1^{\prime} 2^{\prime}$-dihydro- $\beta, \psi$-caroten-4-one) [4], which are $\mathrm{C}_{40}$-type monocyclic carotenoids containing one $\beta$-end group ( $\beta$ ring) (called monocyclic carotenoids in this review). Other marine bacteria including strain P99-3, which belong to the Flavobacteriaceae family, were shown to produce monocyclic carotenoids, myxol $\left(\left(3 R, 2^{\prime} S\right)-3^{\prime}, 4^{\prime}\right.$-didehydro-1',2'-dihydro$\beta, \psi$-carotene-3, $1^{\prime}, 2^{\prime}$-triol $)$ and saproxanthin $\left((3 R)-3^{\prime}, 4^{\prime}\right.$-didehydro- $1^{\prime}, 2^{\prime}$-dihydro- $\beta, \psi$-carotene-3, $1^{\prime}$-diol $)$, and zeaxanthin $\left(\left(3 R, 3^{\prime} R\right)-\beta, \beta\right.$-carotene-3,3'-diol) [5,6], which are a $\mathrm{C}_{40}$-type dicyclic carotenoid containing two $\beta$-end groups (called dicyclic carotenids in this review). Marine bacteria belonging to genus Paracoccus, Brevundimonas or Erythrobacter in the $\alpha$-Proteobacteria class have been revealed to synthesize dicyclic carotenoids that are ketolated at the 4(4')-position(s) (called ketocarotenoids), e.g., astaxanthin $\left(\left(3 S, 3^{\prime} S\right)-3,3^{\prime}\right.$-dihydroxy- $\beta, \beta$-carotene-4,4'-dione) and adonixanthin $\left(\left(3 S, 3^{\prime} R\right)-3,3^{\prime}\right.$ dyhydroxy- $\beta, \beta$-caroten-4-one) (Figure 1) [7-9].

Figure 1. Chemical structures of ketocarotenoids produced in marine bacteria, Paracoccus sp. and Brevundimonas sp., and feasible functions of the carotenoid biosynthesis enzymes. These bacteria synthesize dicyclic carotenoids. Paracoccus sp. and Brevundimonas sp. are demonstrated to possess the unique genes $\operatorname{crt} X$ and $\operatorname{crt} G$, respectively, in addition to the common genes, $\operatorname{crtE}, \operatorname{crtB}, \operatorname{crtI}, \operatorname{crt} Y, \operatorname{crt} Z$, and $\operatorname{crt} W[10,11]$.

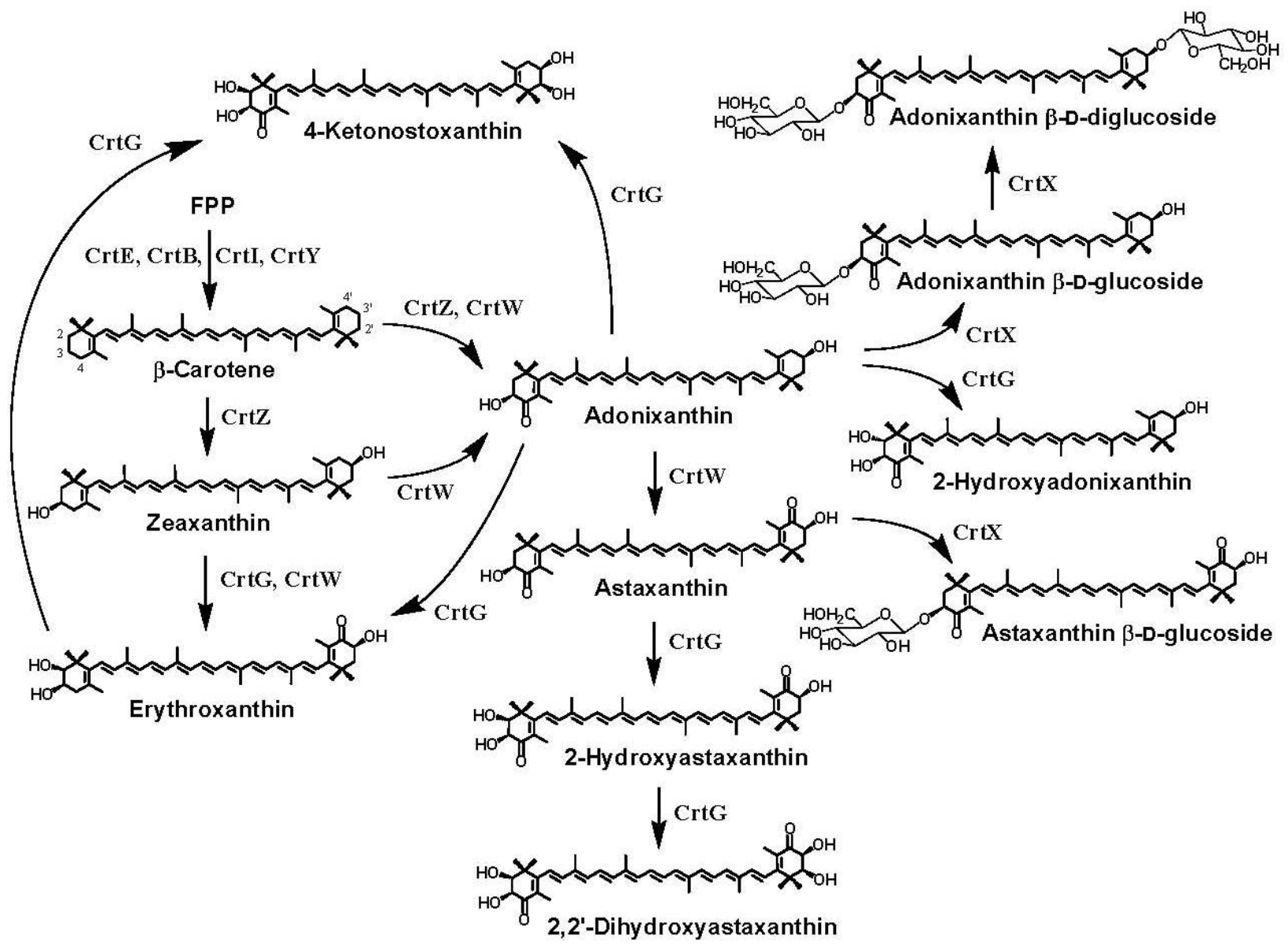


Among ketocarotenoids, astaxanthin and canthaxanthin ( $\beta, \beta$-carotene-4,4'-dione) (specifically the former), are commercially important pigments as nutraceuticals and cosmetics that have anti-oxidation and anti-aging effects as well as colorants in aquaculture, while other ketocarotenoids are likely to have industrial potentials [12-16]. This review focuses on carotenoid $\beta$-ring 4(4')-ketolase (4-oxygenase), carotenoid $\beta$-ring 3(3')-hydroxylase, and carotenoid $\beta$-ring $2\left(2^{\prime}\right)$-hydroxylase, derived from the marine bacteria that belong to $\alpha$-Proteobacteria, and pathway engineering for the production of functional xanthophylls via the incorporation of these $\beta$-ring-modifying enzyme genes.

\section{Bacterial Strains Producing Ketocarotenoids}

Paracoccus sp. strain N81106 (NBRC 101723), isolated from surface seawater near Aka island, Okinawa, Japan, was first shown to produce astaxanthin in bacteria [7,17]. This bacterium was also found to synthesize adonixanthin, adonixanthin $\beta$-D-glucoside, and astaxanthin $\beta$-D-glucoside [7,18]. Paracoccus haeundaesis $\mathrm{BC} 74171^{\mathrm{T}}$, isolated from the Haeundae Coast, Korea, was shown to produce astaxanthin mainly [19]. Paracoccus marinus KKL-A5 ${ }^{\mathrm{T}}$ (NBRC 100637 ${ }^{\mathrm{T}}$ ), isolated from coastal seawater in Tokyo Bay, Japan, was found to produce adonixanthin diglucoside predominantly [20,21]. On the other hands, a marine bacterium Brevundimonas sp. strain SD212 (NBRC 101024) was revealed to synthesize not only astaxanthin and adonixanthin but also their 2(2')-hydroxylated metabolites, that is, 2-hydroxyastaxanthin $\left(\left(2 R, 3 S, 3^{\prime} S\right)-2,3,3^{\prime}\right.$-trihydroxy- $\beta, \beta$-carotene- $4,4^{\prime}$-dione $)$, 2-hydroxyadonixanthin $\left(\left(2 R, 3 S, 3^{\prime} R\right)-2,3,3^{\prime}\right.$-trihydroxy- $\beta, \beta$-caroten-4-one), erythroxanthin $\left(\left(3 S, 2^{\prime} R, 3^{\prime} R\right)\right.$ $3,2^{\prime}, 3^{\prime}$-trihydroxy- $\beta, \beta$-caroten-4-one $), \quad 4$-ketonostoxanthin $\quad\left(\left(2 R, 3 S, 2^{\prime} R, 3^{\prime} R\right)-2,3,2^{\prime}, 3^{\prime}\right.$-tetrahydroxy- $\beta, \beta$ caroten-4-one $)$ and $2,2^{\prime}$-dyhydroxyastaxanthin $\left(\left(2 R, 3 S, 2^{\prime} R, 3^{\prime} S\right)-2,3,2^{\prime}, 3^{\prime}\right.$-tetrahydroxy- $\beta, \beta$-carotene-4,4'dione) [9]. Figure 1 shows the structures of the ketocarotenoids shown in this section and their feasible biosynthetic pathway. Figure 2 shows phylogenetic tree of the marine bacteria that produce astaxanthin and other ketocarotenoids, which were isolated in Marine biotechnology Institute (Kamaishi, Japan), along with the type strains relative to these bacteria, many of which are not marine bacteria but soil bacteria. Interestingly, the phylogenetically closest strains to the marine bacteria Paracoccus sp. N81106 and Brevundimonas sp. SD212 were soil bacteria Paracoccus marcusii DSM $11574^{\mathrm{T}}$ [22] and Brevundimonas aurantiaca ATCC $15266^{\mathrm{T}}$, respectively (Figure 2).

\section{Ketocarotenoid Biosynthesis Genes}

Genes required for the biosynthesis of dicyclic carotenoids were first isolated from soil bacteria Pantoea ananatis (reclassified from Erwinia uredovora) [23] and Pantoea agglomerans (Erwinia herbicola) [24,25], which cannot produce ketocarotenoids and belong to the Enterobacteriaceae family of class $\gamma$-Proteobacteria (the same family to Escherichia coli). The Pantoea carotenoid biosynthesis genes composed a gene cluster for the synthesis of zeaxanthin $\beta$-D-diglucoside from farnesyl diphosphate (farnesyl pyrophosphate; FPP) [25-27], and comprised six genes that encode geranygeranyl diphosphate (GGPP) synthase (CrtE) [27,28], phytoene synthase (CrtB) [27,29], phytoene desaturase (CrtI) [23,30], lycopene ( $\psi, \psi$-carotene) $\beta$-cyclase (CrtY) [23,31], $\beta$-carotene ( $\beta, \beta$-carotene) 3-hydrocylase (CrtZ) [23], and zeaxanthin glucosyltransferase (CrtX) [23,32] (Figure 3). 
Figure 2. Phylogenetic positions of Paracoccus sp., Erythrobacer sp., and Brevundimonas sp. strains deduced from their 16S rRNA sequences. 0 represents marine bacteria. Bacterial strains, whose carotenoid biosynthesis genes were elucidated, are shown in boldface, and the second accession numbers in the parentheses shows those of carotenoid biosynthesis genes. Paracoccus sp. strain N81106 (MBIC01143 = NBRC 101723) and Paracoccus sp. strain PC1 (MBIC03024 = NBRC 101025) were formerly classified as Agrobacterium aurantiacum [17] and Alacaligenes sp. PC-1 [33], respectively. The phylogenetic tree was constructed as described [10]. The scale bar indicates a genetic distance of 0.02 (Knиc).

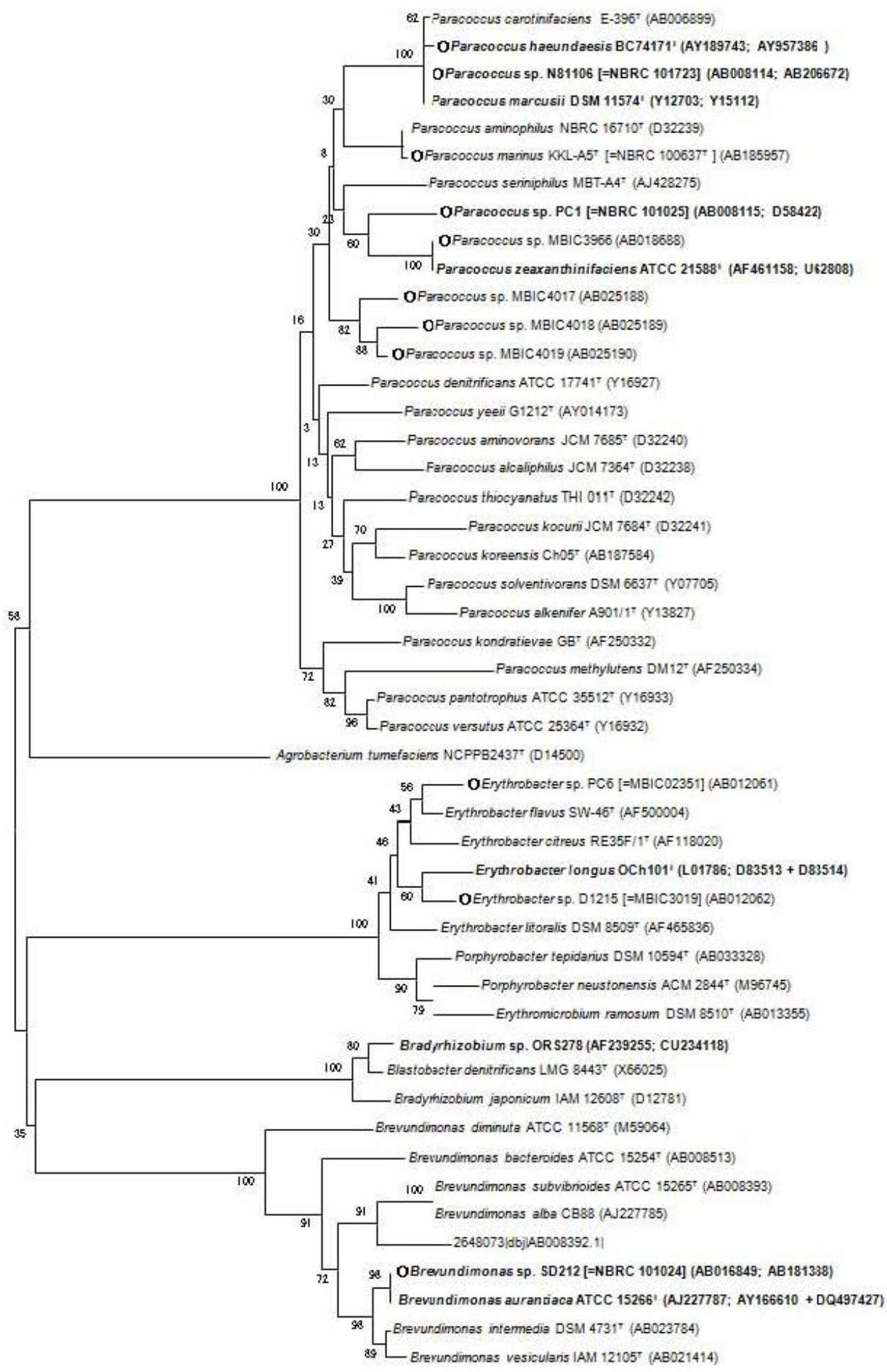


Figure 3. Pathway engineering for the production of functional xanthophylls using the carotenoid biosynthesis genes, $\operatorname{crt} W, \operatorname{crt} Z$, and/or $\operatorname{crt} G$, which were isolated from the marine bacteria, Paracoccus sp. strain N81106 or Brevundimonas sp. strain SD212, in addition to the $\operatorname{crtE}, \mathrm{crtB}, \mathrm{crtI}$, and $\operatorname{crt} Y$ genes (and $\operatorname{crt} X$ ) from $P$. ananatis.

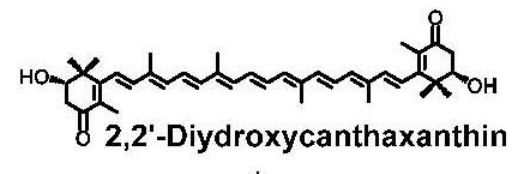

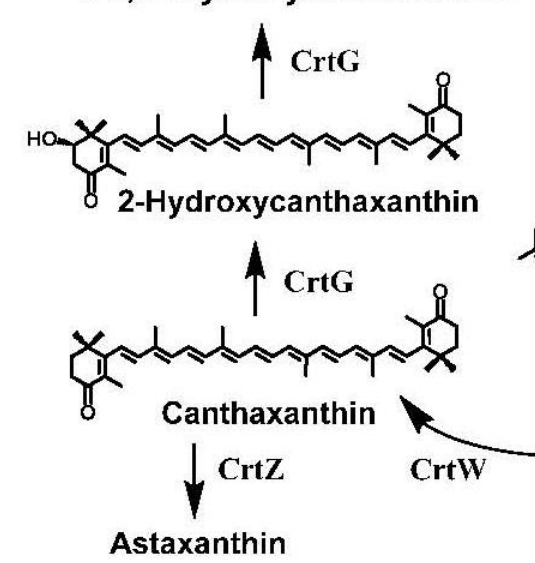

denerech $\mathrm{CH}_{2} \mathrm{OPP}$

FPP

CrtE, $\mid$ CrtB, CrtI

$\checkmark$

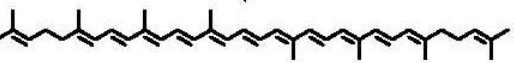

Lycopene

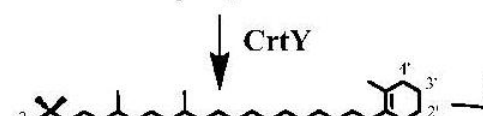

$2^{3}$

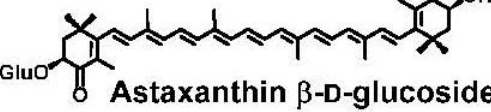

Astaxanthin $\beta$-D-glucoside

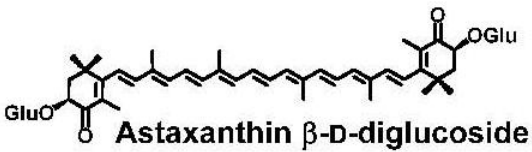

$\uparrow \mathrm{CrtX}$

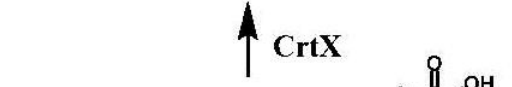

Crtw 4 Crtx

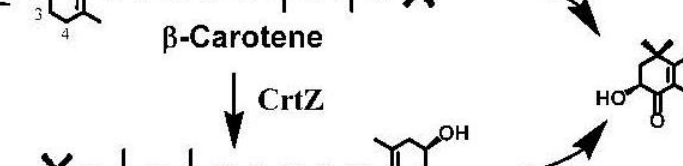

$\downarrow$ CrtG

Zeaxanthin $\beta$-D-diglucoside
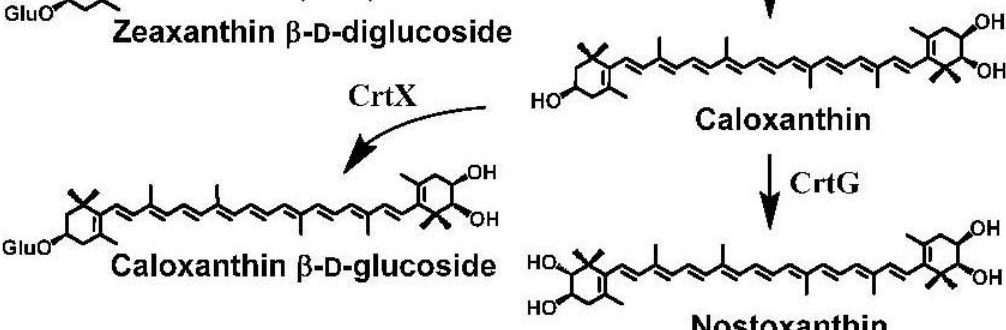

Nostoxanthin

These $c r t$ genes have widely used for complementation analysis of carotenoid biosynthesis genes isolated from other organisms, since they are functionally expressed in E. coli with ease [11,34-37]. The $P$. agglomerans gene cluster contained a gene encoding isopentenyl diphosphate (IPP) isomerase (Idi; type 2) [38] in addition to the six crt genes [39]. These seven carotenogenic (carotenoid-biosynthetic) genes were also found to exist in a carotenoid biosynthesis gene cluster of Paracoccus sp. strain N81106 [10,39]. This cluster included an additional gene, designated CrtW, which was elucidated to code for an enzyme responsible for ketocarotenoid formation, that is, CrtW proved to catalyze the synthesis of canthaxanthin from $\beta$-carotene by complementation analysis using recombinant $E$. coli cells that contains the P. ananatis crtE, crtB, crtI, and $\operatorname{crt} Y$ genes [33] (Figure 3). The hydropathy and transmembrane prediction analyses indicated that $\mathrm{CrtW}$ from Paracoccus sp. N81106 contains four transmembrane domains and two other hydrophobic regions, and its topology model is very similar to those for fatty acid desaturases [40]. It should be noted that it is recalcitrant to purify active $\mathrm{CrtW}$ and $\mathrm{CrtZ}$ proteins, which both are very likely iron-dependent integral membrane proteins, from the recombinant hosts as well as the native hosts, precluding their close enzymatic characterizations. 


\section{Carotenoid 4,4'-Ketolase}

It has been revealed that only two enzymes, carotenoid 4,4'-ketolase $\left(4,4^{\prime}\right.$-oxygenase) ( $\beta$-ring $4\left(4^{\prime}\right)$-ketolase; CrtW) and carotenoid 3,3'-hydroxylase ( $\beta$-ring 3(3')-hydroxylase; CrtZ), are sufficient to biosynthesize astaxanthin from $\beta$-carotene via eight intermediates including zeaxanthin, canthaxanthin and adonixanthin $[35,40,41]$. CrtW can convert not only the (un-substituted) $\beta$ ring but also the 3-hydroxylated $\beta$ ring into the respective 4-ketolated groups, and $\mathrm{CrtZ}$ can convert not only the (un-substituted) $\beta$ ring but also the 4-ketolated $\beta$-ring into the respective 3-hydroxylated groups, as shown in Figure 4 [42-46]. An in vitro analysis with the crude enzymes of $\mathrm{CrtW}$ and $\mathrm{CrtZ}$ from the E. coli cells expressing the corresponding genes indicated that these enzymes are likely 2-oxoglutarate ( $\alpha$-ketoglutarate)-dependent dioxygenases [42].

Figure 4. Catalytic functions of carotenoid 4,4'-ketolases (oxygenases) and carotenoid 3'3'-hydroxylases. BKT means BKT1 or BKT2 from H. pluvialis.
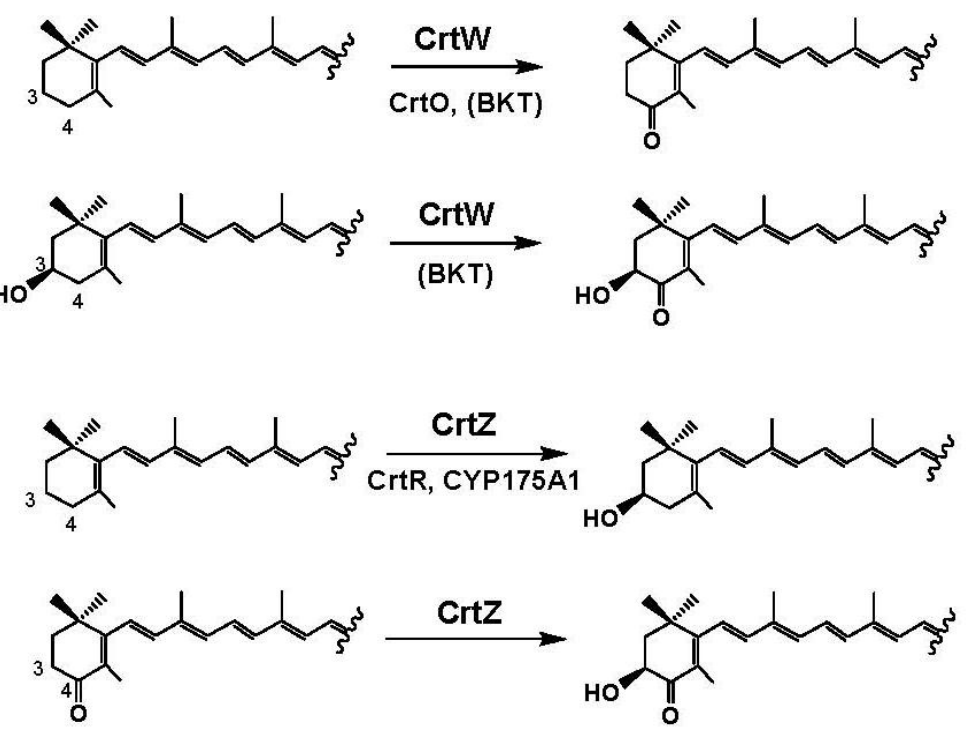

The $c r t W$ genes were present not only in the above-mentioned $\alpha$-Proteobacteria (Figure 2 ) but also in the marine bacterium Algoriphagus sp. KK10202C [4] and cyanobacterial strains such as Anabaena (Nostoc) sp. PCC 7120 and N. punctiforme [47,48]. These cyanobacteria produced not astaxanthin but echinenone ( $\beta, \beta$-caroten-4-one), and 4-ketomyxol 2'-fucoside, a monocyclic carotenoid that includes the 4-ketolated $\beta$-ring [49]. Conversion efficiency to astaxanthin in several CrtWs was compared with recombinant $E$. coli cells that synthesize the carotenoid substrate zeaxanthin due to the presence of the $P$. ananatis crtE, crtB, crtI, crtY, and $c r t Z$ genes, in which each $c r t W$ gene from Paracoccus sp. N81106, Paracoccus sp. PC1, Brevundimonas sp. SD212, Anabaena sp. PCC7120, and $N$. punctiforme was expressed $[44,46]$. It was consequently shown that the Brevundimonas sp. SD212 CrtW, which exhibited the highest amino acid identity (96.3\%) with that of the B. aurantiaca ATCC $15266 \mathrm{CrtW}$ (accession no. AY166610), converted $\beta$-carotene to astaxanthin with the highest efficiency, along with the P. ananatis CrtZ [44,46]. In the case of the Paracoccus CrtWs, not only astaxanthin but also adonixanthin tended to accumulate, and this intermediate was difficult to be converted to astaxanthin $[43,44]$. The cyanobacterial CrtWs poorly converted zeaxanthin to astaxanthin via adonixanthin [46]. 
Two paralogous genes exhibiting significant homology to $c r t W$ were isolated from $H$. pluvialis, and designated $b k t$ [50] or $c r t O$ [51]. These genes were renamed $b k t 1$ from $c r t O$ and $b k t 2$ from $b k t$, since "crtO" has been used for the other type of cyanobacterial $\beta$-ring 4(4')-ketolase genes, as shown later [52]. The BKT1 and BKT2 enzymes are very likely to have catalytic function same to the Paracoccus (or Brevundimonas) CrtWs, considering results from the in vitro study on BKT2 with E. coli [42] and pathway engineering researches in higher plants as well as $E$. coli as the hosts $[16,50,51,53]$.

A gene encoding a new type of $\beta$-ring $4\left(4^{\prime}\right)$-ketolase (named $\mathrm{CrtO}$ ) that showed apparent homology not to CrtW-type ketolase but to CrtI-type phytoene desaturase was first found in cyanobacterium Synechocystis sp. strain PCC 6803 [54], which produced 3'-hydroxyechinenone (3'-hydroxy- $\beta, \beta$-caroten-4-one), zeaxanthin and myxol 2'-dimethyl-fucoside [55]. The $\mathrm{crt} O$ genes were also present in Anabaena sp. PCC 7120 [48], and an actinomycete Rhodococcus erythropolis and Deinococcus radiodurans R1 highly resistant to $\gamma$ and UV radiation [56], which produced other monocyclic carotenoids, e.g., the latter strain produced deinoxanthin (2,1'-dihydroxy-3',4'-didehydro$1 ' 2^{\prime}$-dihydro- $\beta$, $\psi$-caroten-4-one) [1]. An in vivo analysis on $\operatorname{crt} O$ was performed with recombinant $E$. coli cells that synthesize the carotenoid substrate $\beta$-carotene or zeaxanthin, into which each $\mathrm{crt} O$ gene from Synechocystis sp. PCC 6803 and R. erythropolis was introduced and expressed there [57]. This result along with previous finding [48] suggested that the CrtO-type of $\beta$-ring 4(4')-ketolases can accept only the (un-substituted) $\beta$ ring(s) in $\beta$-carotene and probably in monocyclic carotenoids as the substrates (Figure 4).

\section{Carotenoid 3,3'-Hydroxylase}

The crtZ genes have been found not only in carotenogenic bacteria belonging to genera Pantoea, Paracoccus and Brevundimonas, but also in those belonging to the Flavobacteriaceae family [6,39]. Conversion efficiency to astaxanthin in several CrtZs was compared with recombinant $E$. coli cells that synthesize the carotenoid substrate canthaxanthin due to the presence of the P. ananatis crtE, crtB, crtI and $c r t Y$ gens, and the Paracoccus $\mathrm{N} 81106 \mathrm{crtW}$ gene, into which each $\mathrm{crt} Z$ gene from $P$. ananatis, Paracoccus sp. N81106, Paracoccus sp. PC1, Brevundimonas sp. SD212, and marine bacterium strain P99-3 of the Flavobacteriacea family was introduced and expressed there [45]. It was consequently shown that the CrtZ enzymes from Brevundimonas sp. SD212 and the bacterial strain P99-3 converted $\beta$-carotene to astaxanthin with the highest and lowest efficiency, respectively, along with the Paracoccus N81106 CrtW [45].

On the other hands, no crt $Z$ sequences have not been found in cyanobacteria, instead genes encoding a new type of $\beta$-ring 3(3')-hydroxylases (named CrtR) that exhibited moderate homology to $\mathrm{CrtW}$ have been found there [58,59]. The crtR genes were isolated from Synechocystis sp. strain PCC 6803, Anabaena sp. PCC 7120, Anabaena variabilis, and N. punctiforme [46,58]. An in vivo analysis on $c r t R$ was performed with recombinant $E$. coli cells that synthesize the carotenoid substrate $\beta$-carotene or canthaxanthin, into which each $c r t R$ gene from Synechocystis sp. PCC 6803, Anabaena sp. PCC 7120, and A. variabilis was introduced and expressed there [46]. This result along with another result [60] indicated that the CrtR-type enzymes can hydroxylate the (un-substituted) $\beta$ ring of monocyclic carotenoids such as deoxymyxol and deoxymyxol 2 '-fucoside at the 3 position (Figure 4). Among them, only the Synechocystis sp. PCC 6803CrtR was able to convert $\beta$-carotene to 
zeaxanthin [46,58,60]. A thermophilic bacterium Thermus thermophilus HB27, which grows at temperatures above $75{ }^{\circ} \mathrm{C}$, was found to possess another new type of $\beta$-ring 3(3')-hydroxylase of the cytochrome P450 superfamily, named CYP175A1 [61]. The in vivo analysis with the gene strongly suggested that this thermostable P450 accepts only the (un-substituted) $\beta$ ring of $\beta$-carotene as the substrate to form zeaxanthin $[45,61]$.

\section{Carotenoid 2,2'-Hydroxylase}

Carotenoid 2,2'-hydroxylase ( $\beta$-ring 2(2')-hydroxylase) was first found in the marine bacterium Brevundimonas sp. strain SD212, and named CrtG [11]. An in vivo analysis on crtG was performed with recombinant $E$. coli cells that synthesize each carotenoid substrate ( $\beta$-carotene, zeaxanthin, canthaxanthin, or astaxanthin), into which the $\operatorname{crt} G$ gene was introduced and expressed there [11]. The result indicated that the $\mathrm{CrtG}$ can hydroxylate the $\beta$ rings substituted with 3-hydroxy and/or 4-keto groups in dicyclic carotenoids at the 2(2')-positions (Figures 1 and 3) [11]. The crtG genes were also isolated from soil bacteria Brevundimonas vesicularis DC263 and B. aurantiaca ATCC 15266 [62]. The in vivo analysis with these genes indicated that the $B$. aurantiaca $\mathrm{CrtG}$ enzyme (accession no. DQ497427), which exhibited the highest amino acid identity (98.8\%) to that of the Brevundimonas SD212 CrtG, accepted the (un-substituted) $\beta$ rings of $\beta$-carotene in addition to the substituted $\beta$ rings as the substrates [62]. A crtG gene sequence, whose encoded amino acid sequence was $41 \%$ identical to the Brevundimonas sp. SD212 CrtG, was found in a thermophilic cyanobacterium Thermosynechococcus elongatus, which synthesized 2-hydroxylated carotenoids such as caloxanthin $\left(\left(2 R, 3 R, 3^{\prime} R\right)-\beta, \beta\right.$-carotene-2,3,3'-triol $), \quad$ nostoxanthin $\quad\left(\left(2 R, 3 R, 2^{\prime} R, 3^{\prime} R\right)-\beta, \beta\right.$-carotene-2,3, $2^{\prime}, 3^{\prime}$-tetrol $)$ (Figure 3), and 2-hydroxymyxol 2'-fucoside [63].

\section{Pathway Engineering for the Synthesis of Functional Xanthophylls via the Incorporation of crtW, $\operatorname{crt} Z$, and/or $\operatorname{crt} G$ Genes}

Figure 3 shows xanthophylls that were produced in recombinant $E$. coli cells via the incorporation of the marine bacterial $c r t W, c r t Z$, and/or $\operatorname{crt} G$ genes along with the Pantoea crtE, crtB, crtI, and $\operatorname{crt} Y$ genes. The recombinant $E$. coli strain that expresses the four Pantoea crt genes can produce $\beta$-carotene predominantly (approximately $0.2-1 \mathrm{mg} \cdot \mathrm{g}^{-1}$ dry cell weight). The coexpression of the $\operatorname{crt} W, \operatorname{crt} Z$, and/or $\operatorname{crt} G$ genes in the $\beta$-carotene-synthesizing $E$. coli cells confer the ability to produce not only commercially important xanthophylls such as astaxanthin but also xanthophylls minor in nature (e.g., $\beta$-ring(s)-2(2')-hydroxylated carotenoids), which are difficult to synthesize chemically. Particularly, the chemical synthesis of $2\left(2^{\prime}\right)$-hydroxycarotenoids are likely to be recalcitrant, due to high-density around the 1,2-positions of the $\beta$ ring in these xanthophylls. We showed that the coexpression of the Brevundimonas sp. SD212 crtW gene and the $P$. ananatis crt $Z$ gene in the $\beta$-carotene-synthesizing $E$. coli due to the presence of the four crt genes of $P$. ananatis resulted in predominant production of astaxanthin [44,46]. The Paracoccus sp. N81106 crtW gene was evolved by random mutagenesis to have improved activity [40]. It is also demonstrated that the coexpression of the $c r t W$ gene and the $c r t G$ gene from Brevundimonas sp. SD212 or from B. aurantiaca ATCC 15266 in the $\beta$-carotene-synthesizing E. coli resulted in dominant production of 2,2'-dihydroxycanthaxanthin and 2-hydroxycanthaxanthin, while the substrate canthaxanthin accumulated $[11,62]$. The coexpression of the $\operatorname{crt} Z$ gene and the $\operatorname{crt} G$ 
gene in the $\beta$-carotene-synthesizing $E$. coli resulted in predominant production of nostoxanthin along with small amounts of caloxanthin [11,62]. The coexpression of all the three genes $(\operatorname{crt} W, \operatorname{crt} Z$, and $\operatorname{crt} G$ ) in the $\beta$-carotene-synthesizing $E$. coli resulted in dominant production of 2,2'-dihydroxyastaxanthin and 2-hydroxyastaxanthin [11]. When the $P$. ananatis $\operatorname{crt} X$ gene was coexpressed in addition to appropriate combinations of the above $c r t$ genes in $E$. coli, resultant $E$. coli cells were able to synthesize carotenoid-glycosides such as caloxanthin $\beta$-D-glucoside [64] and astaxanthin $\beta$-D-diglucoside [65], as shown in Figure 3.

The $\gamma$-ray-tolerant bacterium $D$. radiodurans $\mathrm{R} 1$ produces the monocyclic carotenoid including the 2-hydroxy-4-keto- $\beta$-ring, deinoxanthin [1]. 2,2'-Dihydroxycanthaxanthin was shown to have strong inhibitory effect against lipid peroxidation in a rat brain homogenate [11]. Such minor ketocarotenoids, which include the 2-hydroxy-4-keto- $\beta$-ring, may have beneficial effects on human health as well as anti-oxidation function, while few works are present examining their biological functions.

When carotenoid biosynthesis genes starting from the utilization of FPP are introduced in E. coli, as above-mentioned, amounts of carotenoids produced with the recombinant $E$. coli cells are far from the practical use, which was difficult to exceed $1 \mathrm{mg} \cdot \mathrm{g}^{-1}$ dry weight. In order to overcome this problem, many pathway engineering researches in $E$. coli have been performed for increasing intracellular concentration of FPP (e.g., recently reviewed [66,67]). For example, the coexpression of the idi (type 1) gene from H. pluvialis, Xanthophyllomyces dendrorhous (renamed from Phaffia rhodozyma), or Saccharomyces cerevisiae, as well as the idi (type 2) from Streptomyces sp. strain CL190, was shown to be effective to increase FPP content $[68,69]$. The introduction of heterologous mevalonate pathway genes in E. coli along with an idi (type 2) gene has been described to efficiently improve the productivity of carotenoids or sesquiterpenes that are synthesized from FPP [69-73]. For example, Yoon et al. [73] produced $22 \mathrm{mg} \cdot \mathrm{g}^{-1}$ dry cell weight of lycopene in $72 \mathrm{~h}$ using such mevalonate-pathway-engineered $E$. coli cells. On the other hand, production of lycopene reached high levels (near to $20 \mathrm{mg} \cdot \mathrm{g}^{-1}$ dry cell weight) in 24-h batch flask culture in pathway-engineered E. coli, which reflected results of multi-dimensional gene target search or gene-knockout analysis [74]. These finding should be applied to efficient production of the above-mentioned functional xanthophylls with E. coli cells.

Pathway engineering researches in higher plants have also been performed for efficient production of astaxanthin, which utilized the marine bacterial crtW genes from Paracoccus sp. N81106 or Brevundimonas sp. SD212, or the $H$. pluvialis bkt1 or bkt2 genes, as reviewed [16,39,53]. For example, the Brevundimonas sp. SD212 crtW and $\mathrm{crtZ}$ genes, whose nucleotide sequence is modified to codon usage of higher plants, were successfully overexpressed in the chloroplasts of tobacco plants (Nicotiana tabacum), and astaxanthin level produce there reached $5.44 \mathrm{mg} \cdot \mathrm{g}^{-1}$ dry weight $(74 \%$ of total carotenoids) [75].

\section{Conclusions}

This review has focused on the carotenoid $\beta$-ring-modifying enzymes, $\mathrm{CrtW}, \mathrm{CrtZ}$ and $\mathrm{CrtG}$, derived from the marine bacteria of the $\alpha$-Proteobacteria class, and pathway engineering for the production of xanthophylls in E. coli, using these enzyme genes. Such pathway engineering researches are performed towards efficient production not only of commercially important xanthophylls such as 
astaxanthin, but also of xanthophylls minor in nature, which are difficult to synthesize chemically, and expected to have beneficial effects on human health as well as anti-oxidation function.

\section{Acknowledgements}

The author gratefully acknowledges the Marine Biotechnology Institute (MBI) that was closed on 30 June 2008, and Kirin Holdings Company, Limited (Kirin Brewery Co., Ltd.). This work was also supported by New Energy and Industrial Technology Development Organization (NEDO) of Japan.

\section{References}

1. Britton, G.; Liaaen-Jensen, S.; Pfander, H. Carotenoids Handbook; Birkhauser Verlag: Basel, Switzerland, 2004.

2. Shindo, K.; Endo, M.; Miyake, Y.; Wakasugi, K.; Morritt, D.; Bramley, P.M.; Fraser, P.D.; Kasai, H.; Misawa, N. Methyl glucosyl-3,4-dehydro-apo-8'-lycopenoate, a novel antioxidative glyco-C30-carotenoic acid produced by a marine bacterium Planococcus maritimus. J. Antibiot. 2008, 61, 729-735.

3. Shindo, K.; Mikami, K.; Tamesada, E.; Takaichi, S.; Adachi, K.; Misawa, N.; Maoka, T. Diapolycopenedioc acid xylosyl ester, a novel glyco- $\mathrm{C}_{30}$-carotenoic acid produced by a new marine bacterium Rubritalea squalenifaciens. Tetrahedron Lett. 2007, 48, 2725-2727.

4. Tao, L.; Yao, H.; Kasai, H.; Misawa, N.; Cheng, Q. A carotenoid synthesis gene cluster from Algoriphagus sp. KK10202C with a novel fusion-type lycopene- $\beta$-cyclase gene. Mol. Genet. Genomics 2006, 276, 79-86.

5. Shindo, K.; Kikuta, K.; Suzuki, A.; Katsuta, A.; Kasai, H.; Yasumoto-Hirose, M.; Matuo, Y.; Misawa, N.; Takaichi, S. Rare carotenoids, (3R)-Saproxanthin and (3R,2'S)-myxol, isolated from novel marine bacteria (Flavobacteriaceae) and their antioxidant activities. Appl. Microbiol. Biotechnol. 2007, 74, 1350-1357.

6. Teramoto, M.; Takaichi, S.; Inomata, Y.; Ikenaga, H.; Misawa, N. Structural and functional analysis of a lycopene $\beta$-monocyclase gene isolated from a unique marine bacterium that produces myxol. FEBS Lett. 2003, 545, 120-126.

7. Yokoyama, A.; Izumida, H.; Miki, W. Production of astaxanthin and 4-ketozeaxanthin by the marine bacterium, Agrobacterium aurantiacum. Biosci. Biotechnol. Biochem. 1994, 58, 1842-1844.

8. Yokoyama, A.; Izumida, H.; Shizuri, Y. New carotenoid sulfates isolated from a marine bacterium. Biosci. Biotechnol. Biochem. 1996, 60, 1877-1878.

9. Yokoyama, A.; Miki, W.; Izumida, H.; Shizuri, Y. New trihydroxy-keto-carotenoids isolated from an astaxanthin-producing marine bacterium. Biosci. Biotechnol. Biochem. 1996, 60, 200-203.

10. Maruyama, T.; Kasai, H.; Choi, S.K.; Ramasamy, A.K.; Inomata, Y.; Misawa, N. Structure of a complete carotenoid biosynthesis gene cluster of marine bacterium Paracoccus sp. strain N81106. Carotenoid Sci. 2007, 11, 50-55.

11. Nishida, Y.; Adachi, K.; Kasai, H.; Shizuri, Y.; Shindo, K.; Sawabe, A.; Komemushi, S.; Miki, W.; Misawa, N. Elucidation of a carotenoid biosynthesis gene cluster encoding a novel enzyme, 2,2'- $\beta$-hydroxylase, from Brevundimonas sp. strain SD212 and combinatorial biosynthesis of new or rare xanthophylls. Appl. Environ. Microbiol. 2005, 71, 4286-4296. 
12. Nishino, H.; Murakoshi, M.; Ii, T.; Takemura, M.; Kuchide, M.; Kanazawa, M.; Mou, X.Y.; Wada, S.; Masuda, M.; Ohsaka, Y.; et al. Carotenoids in cancer chemoprevention. Cancer Metastasis Rev. 2002, 21, 257-264.

13. Pashkow, F.J.; Watumull, D.G.; Campbell, C.L. Astaxanthin: A novel potential treatment for oxidative stress and inflammation in cardiovascular disease. Am. J. Cardiol. 2008, 101, 58D-68D.

14. Camera, E.; Matrofrancesco, A.; Fabbri, C.; Daubrawa, F.; Picardo, M.; Sies, H.; Stahl, W. Astaxanthin, canthaxanthin and $\beta$-carotene differently affect UVA-induced oxidative damage and expression of oxidative stress-responsive enzymes. Exp. Dermatol. 2009, 18, 222-231.

15. Jackson, H.; Braun, C.L.; Ernst, H. The chemistry of novel xanthophyll carotenoids. Am. J. Cardiol. 2008, 101, 50D-57D.

16. Misawa, N. Pathway engineering of plants toward astaxanthin production. Plant Biotechnol. 2009, 26, 93-99.

17. Izumida, H.; Adachi, K.; Nishizima, M.; Endo, M.; Miki, W. Akalone: A novel xanthine oxidase inhibitor produced by the marine bacterium, Agrobacterium aurantiacum sp. nov. J. Mar. Biotechnol. 1995, 2, 115-118.

18. Yokoyama, A.; Adachi, K.; Shizuri, Y. New carotenoid glycosides, astaxanthin glucoside and adonixanthin glucoside, isolated from the astaxanthin-producing marine bacterium, Agrobacterium aurantiacum. J. Nat. Prod. 1995, 58, 1929-1933.

19. Lee, J.H.; Kim, Y.S.; Choi, T.J.; Lee, W.J.; Kim, Y.T. Paracoccus haeundaensis sp. nov., a Gram-negative, halophilic, astaxanthin-producing bacterium. Int. J. Syst. Evol. Microbiol. 2004, 54, 1699-1672.

20. Takaichi, S.; Maoka, T.; Akimoto, N.; Khan, S.T.; Harayama, S. Major carotenoid isolated from Paracoccus schoinia NBRC $100637^{\mathrm{T}}$ is adonixanthin diglucoside. J. Nat. Prod. 2006, 69, 1823-1825.

21. Khan, S.T.; Takaichi, S.; Harayama, S. Paracoccus marinus sp. nov., an adonixanthin diglucoside-producing bacterium isolated from coastal seawater in Tokyo Bay. Int. J. Syst. Evol. Microbiol. 2008, 58, 383-386.

22. Harker, M.; Hirschberg, J.; Oren, A. Paracoccus marcusii sp. nov., an orange gram-negative coccus. Int. J. Syst. Bacteriol. 1998, 48, 543-548.

23. Misawa, N.; Nakagawa, M.; Kobayashi, K.; Yamano, S.; Izawa, Y.; Nakamura, K.; Harashima, K. Elucidation of the Erwinia uredovora carotenoid biosynthetic pathway by functional analysis of gene products expressed in Escherichia coli. J. Bacteriol. 1990, 172, 6704-6712.

24. Armstrong, G.A.; Alberti, M.; Hearst, J.E. Conserved enzymes mediate the early reactions of carotenoid biosynthesis in nonphotosynthetic and photosynthetic prokaryotes. Proc. Natl. Acad. Sci. USA 1990, 87, 9975-9979.

25. Hundle, B.S.; Beyer, P.; Kleinig, H.; Englert, G.; Hearst, J.E. Carotenoids of Erwinia herbicola and an Escherichia coli HB101 strain carrying the Erwinia herbicola carotenoid gene cluster. Photochem. Photobiol. 1991, 54, 89-93.

26. Nakagawa, M.; Misawa, N. Analysis of carotenoid glycosides produced in gram-negative bacteria by introduction of the Erwinia uredovora carotenoid biosynthesis genes. Agric. Biol. Chem. 1991, $55,2147-2148$. 
27. Sandmann, G.; Misawa, N. New functional assignment of the carotenogenic genes $c r t B$ and $c r t E$ with constructs of these genes from Erwinia species. FEMS Microbiol. Lett. 1992, 90, 253-258.

28. Math, S.K.; Hearst, J.E.; Poulter, C.D. The crtE gene in Erwinia herbicola encodes geranylgeranyl diphosphate synthase. Proc. Natl. Acad. Sci. USA 1992, 89, 6761-6764.

29. Neudert, U.; Martines-Ferez, I.M.; Fraser, P.D.; Sandmann, G. Expression of an active phytoene synthease from Erwinia uredovora and biochemical properties of the enzyme. Biochim. Biophys. Acta 1998, 1392, 51-58.

30. Fraser, P.D.; Misawa, N.; Linden, H.; Yamano, S.; Kobayashi, K.; Sandmann, G. Expression in E. coli, purification and reactivation of the recombinant Erwinia uredovora phytoene desturase. J. Biol. Chem. 1992, 267, 19891-19895.

31. Schnurr, G.; Misawa, N.; Sandmann, G. Expression, purification and properties of lycopene cyclase from Erwinia uredovora. Biochem. J. 1996, 315, 869-874.

32. Hundle, B.S.; O’Brien, D.A.; Alberti, M.; Beyer, P.; Hearst, J.E. Functional expression of zeaxanthin glucosyltransferase from Erwinia herbicola and a proposed uridine diphosphate binding site. Proc. Natl. Acad. Sci. USA 1992, 89, 9321-9325.

33. Misawa, N.; Kajiwara, S.; Kondo, K.; Yokoyama, A.; Satomi, Y.; Saito, T.; Miki, W.; Ohtani, T. Canthaxanthin biosynthesis by the conversion of methylene to keto groups in a hydrocarbon $\beta$-carotene by a single gene. Biochem. Biophy. Res. Commun. 1995, 209, 867-876.

34. Misawa, N.; Truesdale, M.R.; Sandmann, G.; Fraser, P.D.; Bird, C.; Schuch, W.; Bramley, P.M. Expression of a tomato cDNA coding for phytoene synthase in Escherichia coli, phytoene formation in vivo and in vitro, and functional analysis of the various truncated gene products. J. Biochem. 1994, 116, 980-985.

35. Misawa, N.; Satomi, Y.; Kondo, K.; Yokoyama, A.; Kajiwara, S.; Saito, T.; Ohtani, T.; Miki, W. Structure and functional analysis of a marine bacterial carotenoid biosynthesis gene cluster and astaxanthin biosynthetic pathway proposed at the gene level. J. Bacteriol. 1995, 177, 6575-6584.

36. Sun, Z.; Gantt, E.; Cunningham, F.X., Jr. Cloning and functional analysis of the $\beta$-carotene hydroxylase of Arabidopsis thaliana. J. Biol. Chem. 1996, 271, 24349-24352.

37. Hannibal, L.; Lorquin, J.; D’Ortoli, N.A.; Garcia, N.; Chaintreuil, C.; Masson-Boivin, C.; Dreyfus, B.; Giraud, E. Isolation and characterization of the canthaxanthin biosynthesis genes from the photosynthetic bacterium Bradyrhizobium sp. strain ORS278. J. Bacteriol. 2000, 182, 3850-3853.

38. Kaneda, K.; Kuzuyama, T.; Takagi, M.; Hayakawa, Y.; Seto, H. An unusual isopentenyl diphosphate isomerase found in the mevalonate pathway gene cluster from Streptomyces sp. strain CL190. Proc. Natl. Acad. Sci. USA 2001, 98, 932-937.

39. Misawa, N. Carotenoids. In Comprehensive Natural Products II Chemistry and Biology; Mander, L., Lui, H.W., Eds.; Elsevier: Oxford, UK, 2010; Volume 1, pp. 733-753.

40. Ye, R.W.; Stead, K.J.; Yao, H.; He, H. Mutational and functional analysis of the $\beta$-carotene ketolase involved in the production of canthaxanthin and astaxanthin. Appl. Environ. Microbiol. 2006, 72, 5829-5837.

41. Sieiro, C.; Poza, M.; de Miguel, T.; Villa, T.G. Genetic basis of microbial carotenogenesis. Int. Microbiol. 2003, 6, 11-16. 
42. Fraser, P.D.; Miura, Y.; Misawa, N. In vitro characterization of astaxanthin biosynthetic enzymes. J. Biol. Chem. 1997, 272, 6128-6135.

43. Fraser, P.D.; Shimada, H.; Misawa, N. Enzymic confirmation of reactions involved in routes to astaxanthin formation, elucidated using a direct substrate in vitro assay. Eur. J. Biochem. 1998, 252, 229-236.

44. Choi, S.K.; Nishida, Y.; Matsuda, S.; Adachi, K.; Kasai, H.; Peng, X.; Komemushi, S.; Miki, W.; Misawa, N. Characterization of $\beta$-carotene ketolases, CrtW, from marine bacteria by complementation analysis in Escherichia coli. Mar. Biotechnol. 2005, 7, 515-522.

45. Choi, S.K.; Matsuda, S.; Hoshino, T.; Peng, X.; Misawa, N. Characterization of bacterial $\beta$-carotene 3,3'-hydroxylases, CrtZ, and P450 in astaxanthin biosynthetic pathway and adonirubin production by gene combination in Escherichia coli. Appl. Microbiol. Biotechnol. 2006, 72, 1238-1246.

46. Makino, T.; Harada, H.; Ikenaga, H.; Matsuda, S.; Takaichi, S.; Shindo, K.; Sandmann, G.; Ogata, T.; Misawa, N. Characterization of cyanobacterial carotenoid ketolase CrtW and hydroxylase CrtR by complementation analysis in Escherichia coli. Plant Cell Physiol. 2008, 49, 1867-1878.

47. Steiger, S.; Sandmann, G. Cloning of two carotenid ketolase genes from Nostoc punctiforme for the heterologous production of canthaxanthin and astaxanthin. Biotechnol. Lett. 2004, 26, 813-817.

48. Mochimaru, M.; Masukawa, H.; Takaichi, S. The cyanobacterium Anabaena sp. PCC 7120 has two distinct $\beta$-carotene ketolases: $\mathrm{CrtO}$ for echinenone and $\mathrm{CrtW}$ for ketomyxol synthesis. FEBS Lett. 2005, 579, 6111-6114.

49. Takaichi, S.; Mochimaru, M.; Maoka, T.; Katoh, H. Myxol and 4-ketomyxol 2'-fucosides, not rhamnosides, from Anabaena sp. PCC 7120 and Nostoc punctiforme PCC 73102, and proposal for the biosynthetic pathway of carotenoids. Plant Cell Physiol. 2005, 46, 497-504.

50. Kajiwara, S.; Kakizono, T.; Saito, T.; Kondo, K.; Ohtani, T.; Nishio, N.; Nagai, S.; Misawa, N. Isolation and functional identification of a novel cDNA for astaxanthin biosynthesis from Haematococcus pluvialis, and astaxanthin synthesis in Escherichia coli. Plant Mol. Biol. 1995, $29,343-352$.

51. Lotan, T.; Hirschberg, J. Cloning and expression in Escherichia coli of the gene encoding $\beta$-C-4-oxygenase, that converts $\beta$-carotene to the ketocarotenoid canthaxanthin in Haematococcus pluvialis. FEBS Lett. 1995, 364, 125-128.

52. Huang, J.C.; Chen, F.; Sandmann, G. Stress-related differential expression of multiple $\beta$-carotene ketolase genes in the unicellular green alga Haematococcus pluvialis. J. Biotechnol. 2006, 122, $176-185$.

53. Giuliano, G.; Tavazza, R.; Diretto, G.; Beyer, P.; Taylor, M.A. Metabolic engineering of carotenoid biosynthesis in plants. Trends Biotechnol. 2008, 26, 139-145.

54. Fernandez-Gonzalez, B.; Sandmann, G.; Vioque, A. A new type of asymmetrically acting $\beta$-carotene ketolase is required for the synthesis of echinenone in the cyanobacterium Synechocystis sp. PCC 6803. J. Biol. Chem. 1997, 272, 9728-9733. 
55. Takaichi, S.; Maoka, T.; Masamoto, K. Myxoxanthophyll in Synechocyctis sp. PCC 6803 is myxol 2'-dimethyl-fucoside, (3R,2'S)-myxol 2'-(2,4-di- $O$-methyl- $\alpha$-L-fucoside), not rhamnoside. Plant Cell Physiol. 2001, 42, 756-762.

56. Tao, L.; Cheng, Q. Novel $\beta$-carotene ketolases from non-photosynthetic bacteria for canthaxanthin synthesis. Mol. Genet. Genomics 2004, 272, 530-537.

57. Choi, S.K.; Harada, H.; Matsuda, S.; Misawa, N. Characterization of two $\beta$-carotene ketolases, $\mathrm{CrtO}$ and $\mathrm{CrtW}$, by complementation analysis in Escherichia coli. Appl. Microbiol. Biotechnol. 2007, 75, 1335-1341.

58. Masamoto, K.; Misawa, N.; Kaneko, T.; Kikuno, R.; Toh, H. $\beta$-carotene hydroxylase gene from the cyanobacterium Synechocystis sp. strain PCC6803. Plant Cell Physiol. 1998, 39, 560-564.

59. Takaichi, S.; Mochimaru, M. Carotenoids and carotenogenesis in cyanobacteria: Unique ketocarotenoids and carotenoid glycosides. Cell. Mol. Life Sci. 2007, 64, 2607-2619.

60. Mochimaru, M.; Masukawa, H.; Maoka, T.; Mohamed, H.E.; Vermaas, W.L.; Takaichi, S. Substrate specificities and availability of fucosyltransferase and $\beta$-carotene hydroxylase for myxol 2'-fucoside synthesis in Anabaena sp. strain PCC 7120 compared with Synechocystis sp. strain PCC 6803. J. Bacteriol. 2008, 190, 6726-6733.

61. Blasco, F.; Kauffmann, I.; Schmid, R.D. CYP175A1 from Thermus thermophilus HB27, the first $\beta$-carotene hydroxylase of the P450 superfamily. Appl. Microbiol. Biotechnol. 2004, 64, 671-674.

62. Tao, L.; Rouvière, P.E.; Cheng, Q. A carotenoid synthesis gene cluster from a non-marine Brevundimonas that synthesizes hydroxylated astaxanthin. Gene 2006, 379, 101-408.

63. Iwai, M.; Maoka, T.; Ikeuchi, M.; Takaichi, S. 2,2'- $\beta$-Hydroxylase (CrtG) is involved in carotenogenesis of both nostoxanthin and 2-hydroxymyxol 2'-fucoside in Thermosynechococcus elongatus strain BP-1. Plant Cell Physiol. 2008, 49, 1678-1687.

64. Osawa, A.; Harada, H.; Choi, S.K.; Misawa, N.; Shindo, K. Production of caloxanthin 3'- $\beta$-D-glucoside, zeaxanthin 3,3'- $\beta$-D-diglucoside, and nostoxanthin in a recombinant Escherichia coli expressing system harboring seven carotenoid biosynthesis genes, including $\operatorname{crt} X$ and $\operatorname{crt} G$. Phytochemistry 2011, 72, 711-716.

65. Yokoyama, A.; Shizuri, Y.; Misawa, N. Production of new carotenoids, astaxanthin glucosides, by Escherichia coli transformants carrying carotenoid biosynthetic genes. Tetrahedron Lett. 1998, 39, 3709-3712.

66. Harada, H.; Misawa, N. Novel approaches and achievements in biosynthesis of functional isoprenoids in Escherichia coli. Appl. Microbiol. Biotechnol. 2009, 84, 1021-1031.

67. Misawa, N. Pathway engineering for functional isoprenoids. Curr. Opin. Biotechnol. 2011, doi:10.1016/j.copbio.2011.01.002.

68. Kajiwara, S.; Fraser, P.D.; Kondo, K.; Misawa, N. Expression of an exogenous isopentenyl diphosphate isomerase gene enhances isoprenoid biosynthesis in Escherichia coli. Biochem. J. 1997, 324, 421-426.

69. Harada, H.; Yu, F.; Okamoto, S.; Kuzuyama, T.; Utsumi, R.; Misawa, N. Efficient synthesis of functional isoprenoids from acetoacetate through metabolic pathway-engineered Escherichia coli. Appl. Microbiol. Biotechnol. 2009, 81, 915-925. 
70. Kakinuma, K.; Dekishima, Y.; Matsushima, Y.; Eguchi, T.; Misawa, N.; Takagi, M.; Kuzuyama, T.; Seto, H. New approach to multiply deuterated isoprenoids using triply engineered Escherichia coli and its potential as a tool for mechanistic enzymology. J. Am. Chem. Soc. 2001, 123, 1238-1239.

71. Newman, J.D.; Marshall, J.; Chang, M.; Nowroozi, F.; Paradise, E.; Pitera, D.; Newman, K.L.; Keasling, J.D. High-level production of amorpha-4,11-diene in a two-phase partitioning bioreactor of metabolically engineered Escherichia coli. Biotechnol. Bioeng. 2006, 95, 684-691.

72. Vadali, R.V.; Fu, Y.; Bennett, G.N.; San, K.Y. Enhanced lycopene productivity by manipulation of carbon flow to isopentenyl diphosphate in Escherichia coli. Biotechnol. Prog. 2005, 21, $1558-1561$.

73. Yoon, S.H.; Lee, Y.M.; Kim, J.E.; Lee, S.H.; Lee, J.H.; Kim, J.Y.; Jung, K.H.; Shin, Y.C.; Keasling, J.D.; Kim, S.W. Enhanced lycopene production in Escherichia coli engineered to synthesize isopentenyl diphosphate and dimethylallyl diphosphate from mevalonate. Biotechnol. Bioeng. 2006, 94, 1025-1032.

74. Alper, H.; Stephanopoulos, G. Uncovering the gene knockout landscape for improved lycopene production in E. coli. Appl. Microbiol. Biotechnol. 2008, 78, 801-810.

75. Hasunuma, T.; Miyazawa, S.; Yoshimura, S.; Shinzaki, Y.; Tomizawa, K.; Shindo, K.; Choi, S.K.; Misawa, N.; Miyake, C. Biosynthesis of astaxanthin in tobacco leaves by transplastomic engineering. Plant J. 2008, 55, 857-868.

Samples Availability: Available from the authors.

(C) 2011 by the authors; licensee MDPI, Basel, Switzerland. This article is an open access article distributed under the terms and conditions of the Creative Commons Attribution license (http://creativecommons.org/licenses/by/3.0/). 